\title{
Customised Care: Post Intensive Care Syndrome
}

\author{
Benno van Tol ${ }^{a}$ Daniëla Dettling ${ }^{b}$ Hinke Kruizenga ${ }^{c}$ Sanne Pellegrom ${ }^{d}$ Mel Major ${ }^{\mathrm{e}}$ \\ Marjolein Siebel $^{f}$ Marike van der Schaaf ${ }^{g}$
}

${ }^{a}$ Amsterdam University of Applied Sciences, Faculty of Health, Department of Physiotherapy, Amsterdam, The Netherlands; ${ }^{b}$ Amsterdam UMC, University of Amsterdam, department of rehabilitation medicine, Amsterdam Movement Sciences, Meibergdreef 9, Amsterdam, The Netherlands; 'Amsterdam University Medical Centers, VU University Amsterdam, department of Nutrition \& Dietetics, de Boelelaan 1117, Amsterdam, The Netherlands; ${ }^{\text {d}}$ Expert Patient representative patient organization IC Connect, Alkmaar, The Netherlands; ${ }^{\text {e European }}$ School Physiotherapy, Amsterdam University of Applied Sciences, Centre of Expertise Urban Vitality, Amsterdam, The Netherlands; ${ }^{f}$ Amsterdam University of Applied Sciences, Department of Occupational Therapy, Amsterdam, The Netherlands; ${ }^{9}$ Centre of Expertise Urban Vitality, Amsterdam University of Applied Sciences, faculty Health, Amsterdam, The Netherlands

\section{Keywords}

COVID-19 · Intensive Care · Malnutrition · PICS · Interdisciplinary collaboration

\begin{abstract}
Physiotherapy, Dietetics and Occupational Therapy have been collaborating over recent years to develop an optimal healthcare programme for patients with Post Intensive Care Syndrome (PICS). This case is an example of PICS symptomatology and focuses on the collaboration between Physiotherapy and Dietetics.
\end{abstract}

What is PICS? Owing to healthcare improvements, more and more patients are surviving the intensive Care Unit (ICU), and recovery during and after ICU stay has been receiving more attention $[1,2]$. Approximately $30 \%$ of the patients admitted to an ICU have persistent symptoms including muscle weakness, reduced walking ability, fatigue, concentration deficits, memory problems, malnutrition, sleep and mood disorders sometimes even years after discharge [3-8]. Since 2012, this combination of physical, cognitive and psychiatric manifestations and reduced quality of life after staying in an ICU has been recognised as Post Intensive Care Syndrome (PICS) [9]. The impact of PICS is often not limited to the patient as it may also impact the mental status of the patient's immediate family. This is known as PICS-Family (PICS-F) [10-12].

Treatment of PICS: Approximately $80 \%$ of PICS patients need pri-

\section{Medical History and Hospital Admission}

An 85-year-old male patient was admitted to hospital with a primary diagnosis of small intestine ileus. The full history included asthma, lung surgery, kidney disease managed with haemodialy- mary care physiotherapy. Physiotherapists and GPs are often the only primary care professionals involved in the recovery process of these patients after hospital discharge [13, 14]. Both patients and healthcare professionals report a number of difficulties, e.g. limited transmural continuity in healthcare, coordination of multidisciplinary activities, supportive treatment guidelines and specific knowledge of pathology, treatment and prognosis. Patients report that they are not adequately supported when resuming their professional activities and that medical and allied healthcare treatments do not fully meet their needs at that time [15-18].

The REACH project: In order to improve the situation, the REACH project (REhabilitation After Critical illness and Hospital discharge) was started in Amsterdam region in the Netherlands. Within REACH, a Community of Practice - consisting of professionals (physiotherapists, occupational therapists, dieticians), those who live or have lived with the condition and researchers - has developed a transmural rehab programme. A special attribute of this programme is the integration of the concept of "positive health". The case in this article describes the treatment of a PICS patient treated within the REACH network.

\section{(c) 2020 NTVD}

https://ntvd.media/artikelen/het-post-intensive-care-syndroom/ sis, Coronary Artery Bypass Graft and digestive tract haemorrhage. Before the hospital admission, the patient was able to climb stairs, walk short distances without help and did not have mental problems or cognitive dysfunction. He lived with his partner in independent housing.

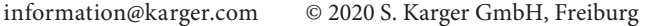
www.karger.com/knd

Karger $\stackrel{\text { ! }}{=}$
Hinke Kruizenga

Amsterdam University Medical Centers

VU University Amsterdam, department of Nutrition \& Dietetics

de Boelelaan 1117

Amsterdam, The Netherlands 


\section{- MY POSITIVE HEALTH}

- Looking after yourself

- Knowing your limitations

- Knowledge of health

- Managing time

- Managing money

- Being able to work

- Asking for help
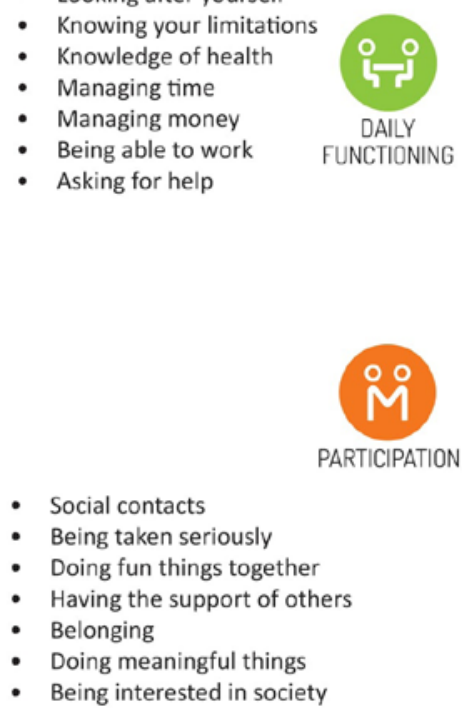

- Being interested in society

Institute for Positive Health (iPH) | Dialogue tool 1.0
- Feeling healthy

- Feeling fit

- Having complaints and/or pain

- Sleeping pattern

- Eating pattern

- Physical condition

- Exercise
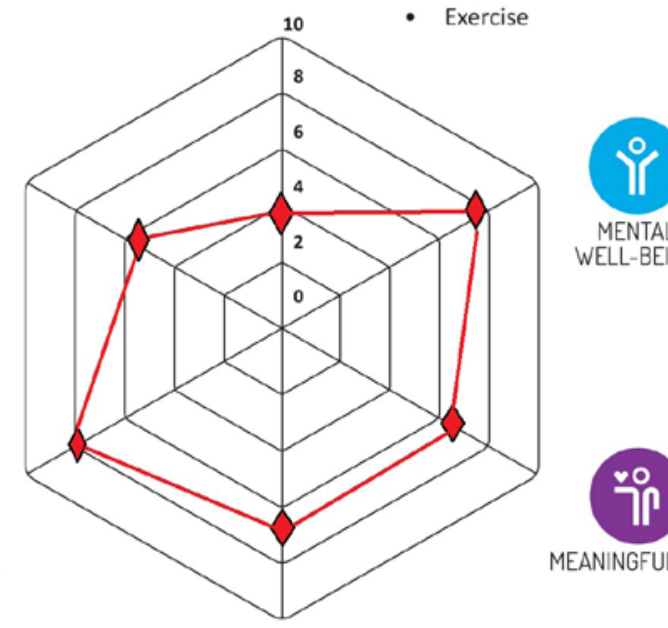

MENTAL

WELL-BEING
- Being able to remember things

- Being able to concentrate

- Being able to communicate

- Being cheerful

- Accepting yourself

- Being able to handle changes

- Having controle

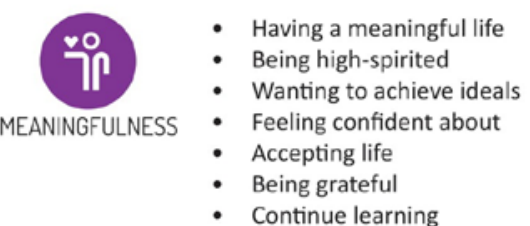

- Enjoyment

- Being happy

- Feeling good

- Feeling well-balanced

- Feeling safe

- Living conditions

- Having enough money

- Continue learning

Fig. 1. Completed 'spiderweb' tool to start the discussion [19].

During the hospital stay, he developed sepsis due to small intestine perforation for which he underwent emergency laparotomy. During a postoperative stay in the ICU, the situation was further complicated by cardiogenic shock, pneumonia and terminal kidney failure. He was mechanically ventilated for two days.

On day 2 postoperatively, the patient started physiotherapy (breathing exercises, bedside exercise therapy, cycling in bed, transfer training) alongside his haemodialysis and medicinal therapy. $\mathrm{He}$ was transferred to the ward on Post-op day 10. Transfer training continued in the ward and he was given gait training using a walker. Dietetics was also involved here; the dietician started an energy and protein dense diet suitable for the patient's limited appetite ensuring adequate protein intake (1.2 to 1.5 grams per kg per day). Physiotherapy, walking aids and homecare (twice daily) were requested at discharge. The dialysis dietician was already involved in the treatment of the patient. The patient was discharged on day 18 postoperative. After discharge, physiotherapy was handed over to a primary care physiotherapist within the REACH network. The handover procedure within this network consists of a discharge letter - geared to patients with PICS - and a telephone call between the hospital therapist and the primary care physiotherapist.

\section{Research}

Within the REACH network, patients are visited by the physiotherapist at home as soon as possible which, in this case, happened six days after discharge. The first step in the process involved identifying the healthcare need the patient had, as well as their health perception from a 'postitve health' viewpoint, from which a spiderweb can be drawn (see figure 1) [19]. The patient felt that his quality of life could be improved, specifically his physical function and that he would like to improve his maximum walking distance.

\section{Physiotherapy}

Physical examination revealed that the patient's overall muscle strength was 4 (Medical Research Council, MRC) and that his grip strength was inadequate ( $22 \mathrm{~kg}$, where $32 \mathrm{~kg}$ was the norm). His inspiratory muscle strength was $-61 \mathrm{~cm} \mathrm{H} 2 \mathrm{O}$ (reference value $=85 \mathrm{~cm} \mathrm{H2O}$ ) [20]. He did not have restricted joint mobility, but did feel stiffness and pain when moving (5 on 10-point scale). Fatigue (measured using the Modified Fatigue Impact scale, MRI) gave a high (maximum) score of 20 points on 'physical fatigue' and 'reduced activity'. The 'reduced motivation' was moderately in- 
creased (14 points) and he scored 'normal' (5 points) on mental fatigue $[21,22]$.

Assistance was needed from one person to transfer from sitting to standing. The maximum walking distance was approximately 100 metres using a wheeled walker taking four breaks.

The patient scored a remarkably high score of 8 on a 10 -point 'health perception' scale for participation. His wife, children and friends were very supportive. His home was adequate for using his wheeled walker safely both inside and outside. The REACH physiotherapist screened for malnutrition using the $\mathrm{SNAQ}^{65+}[23]$. The 'red' score confirmed that the patient had an indication for dietetic care. The screening questions for cognitive function, occupational therapy and the results on the Global Psychotrauma Screen [24] did not suggest that occupational therapy was needed.

\section{Dietetics}

The patient was found to have lost a significant amount of weight, which had already started before the surgery. The patient's body composition (ratio of fat mass to muscle mass) was abnormal. This was attributed to weight loss due to illness and inactivity (Fat Free Mass Index $<$ P5, Fat Mass Index = P25) $[25,26]$. He had little appetite (5 on a 10-point scale), had nausea due to fatigue and consumed only approximately $75 \%$ of his daily energy and protein requirements. Based on the patient's weight loss, intake, functional tests and body composition, the dietician diagnosed malnutrition and sarcopenia $[27,28]$.

\section{Diagnosis}

This patient met the previously described PICS symptomatology. Patients treated in an ICU present with a wide range in pre-admission functional status, primary diagnoses, comorbidities and other factors that may influence burden and coping capacity [29] Known factors that influence the outcome of the patient's recovery programme were: beeing of older age, extensive medical history, unknown baseline situation and good support from the patient's wife and family [12, 30-33]. The significant medical history and unknown preoperative coping capacity made it difficult to estimate the patient's postoperative function and the feasibility of achieving the ultimately desired function. The home visit clarified some of the factors facilitating the patient's recovery, such as the involvement of the wife and family and the home situation which provided an effective and safe environment for exercise therapy. The use of the positive health-concept in this case promoted a shift in focus from the extensiveness and complexity of medical data to the patient himself. The patient's resilience was very important: in this case to improve his mobility (especially independent mobility) [34, 35].
Screening Tools

In the hospital, patients with PICS are treated by multidisciplinary teams. These teams bring together different types of expertise [36, 37]. However, malnutrition and sarcopenia are more commonly the rule than the exception in patients with PICS [23]. REACH physiotherapists use the $\mathrm{SNAQ}^{65+}$ to consider whether a dietician is needed to treat a particular patient.

Simple screening tools to assess cognitive and psychosocial function for use in patients with PICS are not yet available for physiotherapists. The REACH network uses various self-developed screening questions that help to understand the patient's cognitive function and their ability to perform their ADL (to assess the appropriateness of occupational therapy). In this case, the conclusion was that the patient did not have an indication for referral to occupation therapy.

The Global Psychotrauma Screen (GPS) was used to get an impression of common psychological conditions after a stay in an ICU, such as PTSD. This is a simple tool for rough screening of traumarelated psychological prob-lems [24]. The score on this tool did not trigger the need for further psychological assessment.

\section{Treatment Goals}

The patient's goals were to walk independently without walkingaids for a period of 10 minutes, and more generally: to improve his maximum walking distance, nutritional status, to stimulate resilience and ability to self-manage, and finally to involve the patient's wife in the treatment (PICS-F).

\section{Multidisciplinary Treatment}

The physiotherapist visited twice weekly on days the patient did not have dialysis. Exercises included rolling over in bed, getting out of bed, sitting down and walking with the walker. This resulted in significant progress within a few weeks. The walker exercises were expanded from indoors to outdoors. Agreements were made to expand the walking training guided by fatigue while taking the patient's dialysis into consideration. The walker allowed the patient to walk greater distances feeling safer.

The dietician spoke to the patient during his haemodialysis. She worked with the patient and his wife to make some changes to meal timings and diet. Despite his poor appetite and nausea, he managed to consume sufficient calories and proteins, while still taking into consideration the restrictions associated with haemodialysis. The importance of additional protein around periods of physical exercise and protein-rich snacks before going to sleep was highlighted.

The physiotherapist and dietician both reported to the GP. Healthcare evaluation revealed that both were involved, but that there was no coordination. The literature suggests that coordinated intervention of nutrition and exercise training can deliver added value in vulnerable patient groups [38-42]. 


\section{Results}

The patient and physiotherapist were so satisfied with the patient's home-based treatment leading to improved physical function that further treatment in the physiotherapy practice was not needed. After ten weeks, the patient's overall fitness and his ability to use his walker had improved to the extent that he was easily able to walk outside without breaks for twenty minutes. The patient was satisfied and able to maintain or even improve his fitness independently. Walking independently without a walker was no longer considered feasible or desirable. The patient was happy to be able to resume his normal activities with his wife. He was able to resume driving together with his wife. The physiotherapy was stopped.

His nutritional status improved slightly as evidenced by his weight gain and increased muscle mass. His intake was adequate, and his appetite and nausea improved. The dietician continued to be involved in the treatment because of the haemodialysis.

Further assessments were made at three and six months after discharge. This confirmed that the patient was able to maintain and even further improve his ability to walk. No PICS-related cognitive dysfunction or mental problems were observed.

Recovery from PICS can take months or even years. New PICSrelated symptoms may also manifest or arise; monitoring should therefore be continued. The major variations in patient characteristics and recovery make it difficult to predict the prognosis and suggest that caution is appropriate.

\section{Learning Points}

The group of PICS patients is very heterogeneous. In this case, the patient's age, extensive medical history and medical records were the reason to suspect the condition. However, the patient eventually made an almost full recovery without complications.

The first contact at home clearly delivered added value in terms of assessing the patient's function and special attention was given to bed mobility and transfers. The home situation and involvement of the patient's wife and acquaintances were also helpful. The patient felt that the assessments of his physical function (strength, fitness) were frustrating and negative, but the concept of positive health helped him rediscover what he was able to do, which led to him finding his resilience and ability to self-manage. An important point to consider is coordination between the dietician, GP and physiotherapist (or the need for this). This did not take place in this case. However, we do not know whether coordinating nutrition and exercise therapy would have delivered added value in this case. Based on the patient's level of functioning and motivation, there were no doubts about his ability to continue to be active. Further follow-up was not performed. The REACH programme offered structure and supported the treatment.

\section{References}

References to this article are available at www.karger.com/doi/10.1159/ 000513300 . 